\title{
Successful Treatment of Corticosteroid-Induced Rosacea-Like Dermatitis with Platelet-Rich Plasma Mesotherapy: Report of Seven Cases
}

\author{
Xing Fan · Yue Yin (D) - Wenjie Dou · Tong Li · Ping Xue • \\ Qing Yang · Qiaoxin Ma
}

Received: December 11, 2020 / Accepted: January 20, 2021 / Published online: February 4, 2021

(C) The Author(s) 2021

\begin{abstract}
Introduction: Corticosteroid-induced rosacealike dermatitis (CIRD) is one of the cutaneous side effects of long and excessive application of topical corticosteroids, resembling rosacea that can present with a series of cutaneous manifestations. Most patients with CIRD undergo a variety of long-term treatments before their symptoms are relieved as there is no accepted standard therapy. We gave each of seven patients two sessions of platelet-rich plasma (PRP) mesotherapy before the routine treatment to restore their skin barrier function, and we were surprised to find that the patients' symptoms and appearance were significantly improved without any further treatments. Here we report this unexpected finding in dealing with CIRD.

Case Presentation: Seven patients, one male and six female, who had history of applying topical corticosteroids on the face for more than 3 months continuously or intermittently for various reasons were diagnosed with CIRD, showing varying degrees of erythema, telangiectasia, and papules, with dry skin, pain, and
\end{abstract}

X. Fan · Y. Yin $(\bowtie) \cdot$ W. Dou - T. Li · P. Xue .

Q. Yang · Q. Ma

Department of Plastic Surgery, Xijing Hospital, The

Fourth Military Medical University, Xi'an 710032,

Shaanxi, China

e-mail: yiny110@sina.com burning or stinging sensation. We intended to use the restorative effect of PRP to restore the skin barrier function of the patients for further treatment such as tacrolimus ointment, intense pulsed light (IPL), or pulsed dye laser (PDL), so they were treated twice with PRP intradermal injections using a mesogun injector machine. We used the VISIA skin detector to photograph and compare the changes after the treatments. Clinical efficacy was also assessed by the Global Aesthetic Improvement Scale (GAIS). Patients' assessments were recorded using three-point scale questionnaires. After two sessions of PRP treatment, before any further treatment, we were surprised to find that the patients' symptoms had improved significantly, as confirmed by VISIA skin detector and GAIS. All of the patients were satisfied with the outcomes, considering that mesotherapy was not painful and their appearance and symptoms had much improved.

Conclusions: PRP mesotherapy may mitigate sensitivity and inflammation in patients with CIRD by reducing erythema, telangiectasia, and papules. It is worth conducting large randomized controlled trials to verify its safety and efficacy in treating corticosteroid-induced rosacea-like dermatitis and maybe rosacea.

Keywords: Corticosteroid-induced rosacea-like dermatitis (CIRD); Intradermal injection; Mesotherapy; Platelet-rich plasma (PRP); Skin barrier 


\section{Key Summary Points}

Corticosteroid-induced rosacea-like dermatitis (CIRD) is one of the cutaneous side effects of long and excessive application of topical corticosteroids, and most of patients with CIRD undergo a variety of long-term treatments before their symptoms are relieved as there is no accepted standard therapy.

We intended to use the restorative effect of platelet-rich plasma (PRP) to restore the skin barrier function of the patients for further treatment such as tacrolimus ointment, intense pulsed light (IPL), or pulsed dye laser (PDL), expecting that they would respond better to these treatments.

After two sessions of PRP treatment, before any further treatment, we were surprised to find that the patients' symptoms had improved significantly, and all of the patients were satisfied with the outcomes.

From the follow-up, the PRP repair effect is relatively lasting. We believe that when the skin barrier is functioning better, the subsequent treatment will respond faster.

On the basis of these seven cases, we consider that it is worth conducting large randomized controlled trials to verify the safety and efficacy of PRP mesotherapy in treating CIRD. Plus, it may also provide new ideas for the treatment of rosacea and other skin disorders associated with impaired skin barrier function.

\section{DIGITAL FEATURES}

This article is published with digital features, including a summary slide, to facilitate understanding of the article. To view digital features for this article go to https://doi.org/10.6084/ m9.figshare.13615841.

\section{INTRODUCTION}

Topical corticosteroids have a major role in the management of many skin diseases. They exert anti-inflammatory, antimitotic, and immunosuppressive effects through a variety of mechanisms. Whereas topical use of corticosteroids is generally safer than systemic application, cutaneous and systemic side effects may develop after long or excessive use. Corticosteroid-induced rosacea-like dermatitis (CIRD) is one of the cutaneous side effects, resembling rosacea that can present with a variety of cutaneous manifestations. Skin involvement primarily affects the face where topical corticosteroids were used for different reasons, with a varying constellation of signs and symptoms such as acneiform eruption, erythema, papules, pustules, facial hot flashes, persistent redness, telangiectasia, pruritus, dryness, pain, and burning or stinging sensation. CIRD starts after long and excessive application of topical corticosteroids, usually as a result of recommendations from nonmedical personnel or through self-prescribing [1]. Many patients seek therapy because of concern over the effect of CIRD on physical appearance, but satisfactory improvement is hard to attain as there is no standard cure for this skin disorder. Current management includes avoidance of flushing [2-4], discontinuation of offending topical steroid [5], gentle skin care practices, sun protection $[6,7]$, intense pulsed light (IPL) [8], pulsed dye laser (PDL) [9], oral and/or topical antibiotics [10], and topical calcineurin antagonists such as tacrolimus or pimecrolimus ointment $[9,11]$.

Platelet-rich plasma (PRP) has been used for healing or regeneration of damaged tissue in many fields. Growth factors within platelets are chemotactic for monocytes, fibroblasts, stem cells, endothelial cells, and osteoblasts and are mitogenic for fibroblasts, smooth muscle cells, osteoblasts, endothelial cells, and keratinocytes, which help initiate repairing of cutaneous barrier function and positive tissue healing responses such as promotion of collagen production [12-14]. We attempted to use this repair effect to restore the skin barrier function in patients with CIRD, some of whom had 
received many other treatments but with disappointing outcomes, for further treatment. Considering that the skin barrier of patients with CIRD has been damaged, we wanted to make PRP play its role fully and not aggravate the patients' skin barrier injury, so we chose to perform mesotherapy to deliver PRP into the superficial dermis. Surprisingly, after two sessions of treatment, we found unexpected significant improvement in both symptoms and appearance in all seven patients. We have obtained consent for publication from each subject with consent forms fully understood and signed by all the participants themselves. Written informed consent was obtained from the patient for publication of this case report and accompanying images.

\section{CASE PRESENTATION}

\section{Patient Information}

The diagnosis was established on a clinical basis (Table 1). A detailed history regarding symptoms, signs of the disease, duration of existing problem, topical steroid used and its duration, and purpose of using steroid were noted down $[1,15,16]$. Seven patients diagnosed with CIRD at our department between December 2016 and August 2018 had a history of topical corticosteroid use on the face for more than 3 months continuously or intermittently for various reasons such as seborrheic dermatitis, eczema, and rashes of unknown origin. There were two cases of daily use for more than 3 months, five cases

Table 1 Clinical findings in patients with CIRD

\begin{tabular}{ll}
\hline Clinical findings & Number of patients \\
\hline Telangiectasia & 7 \\
Dry skin & 6 \\
Erythema & 6 \\
Papules and pustules & 4 \\
Persistent redness & 3 \\
Burning or stinging sensation & 2 \\
\hline
\end{tabular}

of intermittent use, some once a day, some twice a day with the average withdrawal period less than 3 weeks and maximum duration up to 19 months. Their ages ranged between 25 and 60 years and the female to male ratio was 6:1.

\section{PRP Preparation}

We used the specialized PRP preparation set and PRP preparation centrifuge (all produced by Shandong WEGO group medical polymer Co. Ltd, China) to extract PRP. The process begins with whole blood taken from each patient by peripheral venipunctures [17]. First, open the kit, take out the 50-ml syringe and anticoagulant, extract the anticoagulant (the ratio of anticoagulant to blood is 1:9), connect the blood sampling needle, and take $45 \mathrm{ml}$ blood from the patient's central cubital vein. Then inject the blood into centrifuge tube $\mathrm{A}$, balance the centrifuge tube B with normal saline of the same weight, and place the tubes into the centrifuge symmetrically. After the first centrifugation, at $2000 \mathrm{rpm}$ for $10 \mathrm{~min}$, red blood cells in the lower layer of tube $\mathrm{A}$ are extracted and discarded, the appropriate amount of normal saline is extracted from tube B to balance, and the tubes are placed symmetrically in the centrifuge. After the second centrifugation, at $2200 \mathrm{rpm}$ for $10 \mathrm{~min}$, the supernatant on the upper layer of tube A and a small amount of red blood cells in the bottom layer are extracted and discarded, and the remaining middle liquid is PRP [18], which is usually 3-5 $\mathrm{ml}$.

\section{Mesotherapy}

Pretreatment baseline photographs of the full face, the right, and left side of the face and VISIA (the sixth generation, version number 6.3.4, produced by Canfield USA) scans were taken of each patient. Topical anesthesia was applied before treatment. We used a Derma Shine II mesogun injector machine (produced by HUONS, Korea) to carry out the mesotherapy. This machine is equipped with a 9-needle (32 gauge each) mesogun, and each needle delivers a fixed volume of solution into the skin. The mean depth of injections was $1.0 \pm 0.2 \mathrm{~mm}$ 
to make sure that PRP was injected into the superficial layer of the dermis of the skin. PRP is extracted with a special syringe and connected to the needle, and nine pinholes can be produced after one injection. The injections start from the forehead in a row, until the injections are completed in the whole face. After the treatment we applied sterilized facial mask and ice compress over the facial mask for $20 \mathrm{~min}$ to alleviate mild hemorrhage and to reduce the severity of bruising. Daily skin care regimens, including makeup to cover any petechiae or redness, were resumed after $24 \mathrm{~h}$. Every patient received two sessions of mesotherapy at 1-month interval.

\section{Efficacy Assessment}

Clinical efficacy was assessed by VISIA scans after each session and by investigators (doctors who did not perform the mesotherapy) on 30, 60,120 , and 210 days after baseline, using the Global Aesthetic Improvement Scale (GAIS). GAIS was measured for each patient compared to the baseline photographs, with the rating categories of "worse", "no change", "improved", "much improved", and "very much improved" [19]. After the first session (day 30) and at days 60 and 210 after baseline, the patient's assessment was also evaluated through threepoint scale questionnaires (not satisfied,
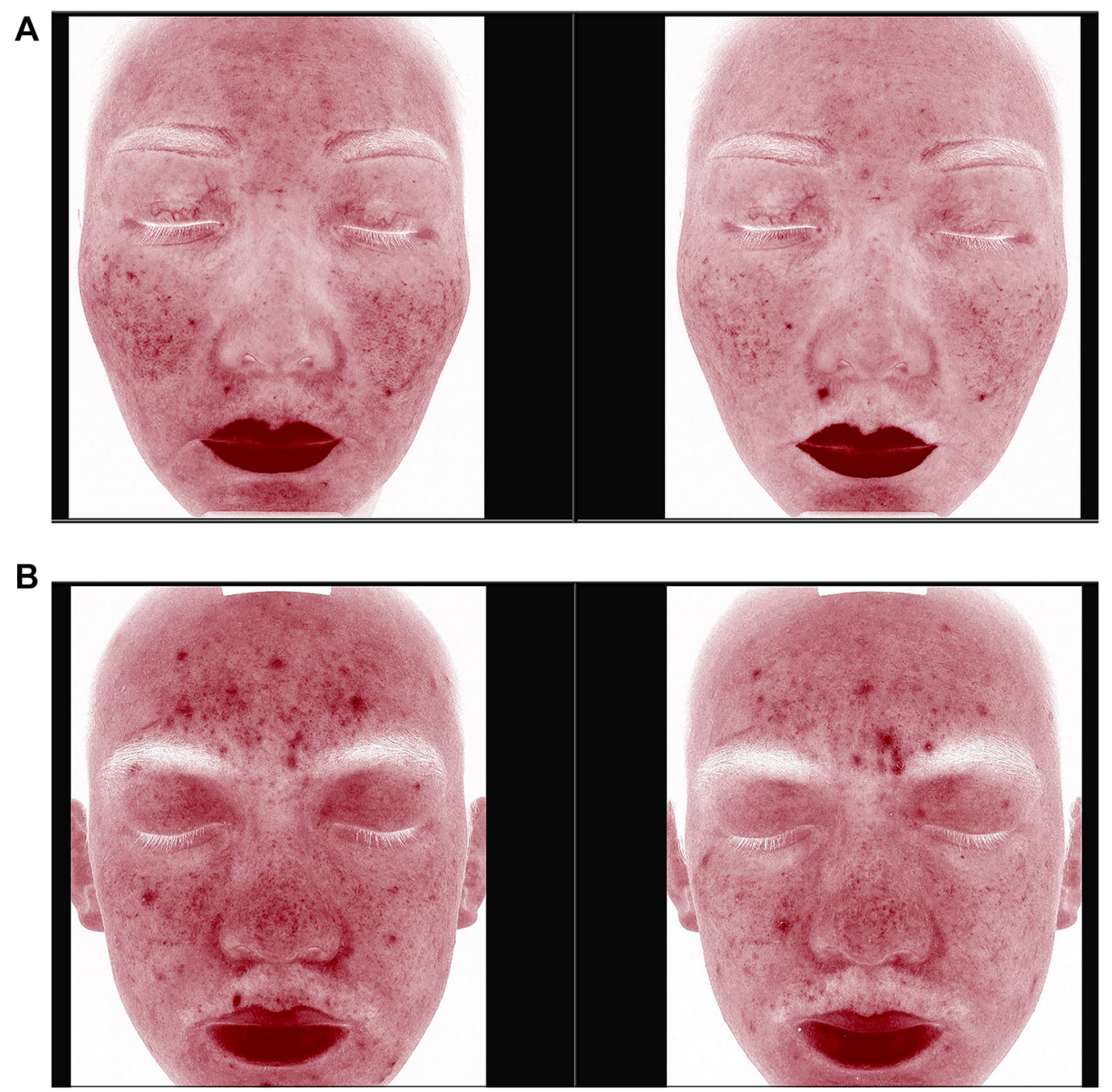

Fig. 1 a Female, 35 years old, telangiectasia had relieved after 2 sessions of mesotherapy. b Male, 27 years old, acneiform eruption had relieved after 2 sessions of mesotherapy 
A
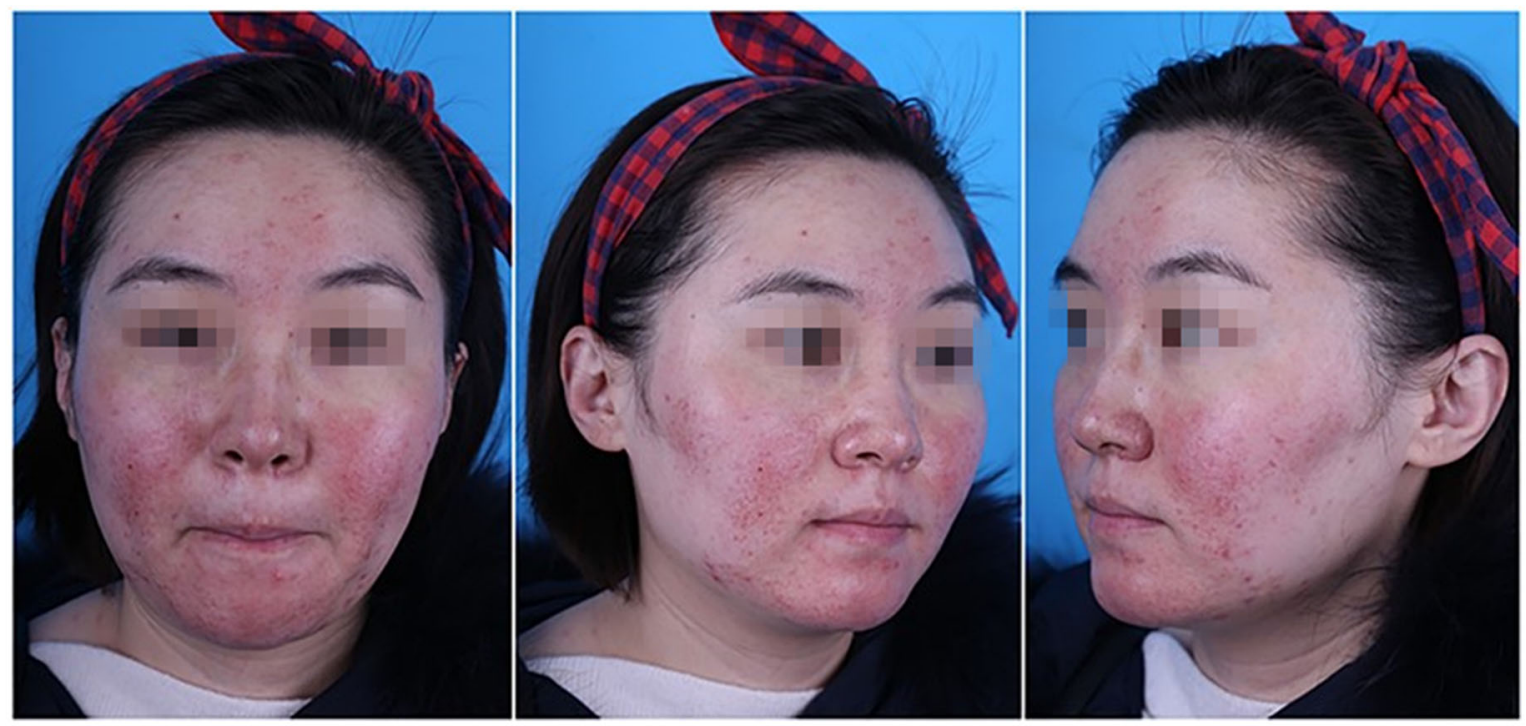

B
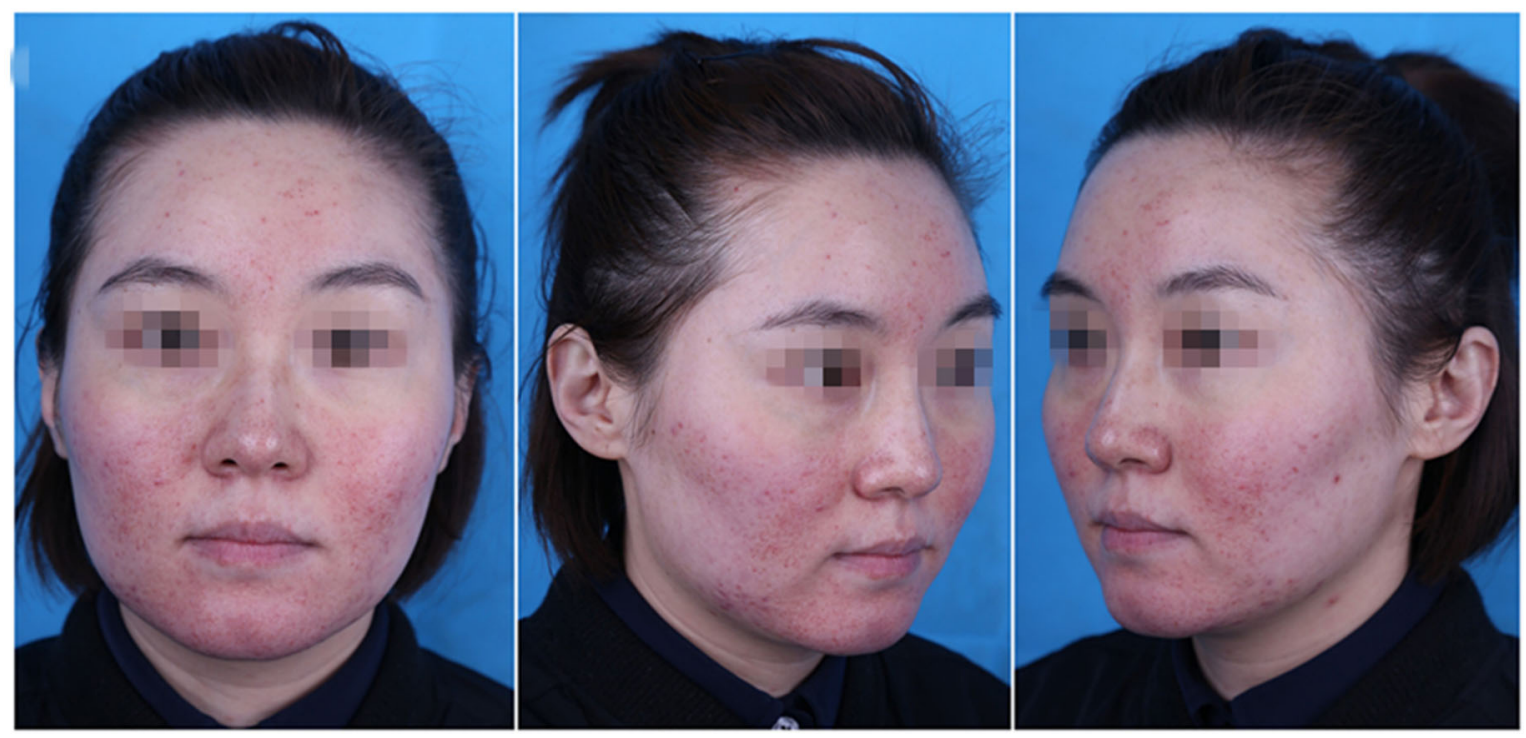

Fig. 2 a Female, 26 years old, redness and telangiectasia were found with dry skin and burning sensation. $\mathbf{b}$ Both appearance and symptoms were improved after 2 sessions of mesotherapy

satisfied, very satisfied) according to their change of appearance and their treatment experiences.

After two sessions of PRP mesotherapy, telangiectasia, erythema, and papules had relieved in all patients compared to the pretreatment photographs according to the VISIA scans (Fig. 1). On the basis of investigator-evaluated GAIS scores, rosacea-like appearance had improved in five patients at day 30 and in all seven patients at day 60 , and the efficacy lasted till day 210 (Fig. 2). Investigators judged the rosacea-like appearance as "much improved" for six patients at day 120 compared to baseline (Table 2).

All patients were satisfied with the treatment, indicating that the mesotherapy was painless or only slightly painful, and the 
Table 2 Global Aesthetic Improvement Scale

\begin{tabular}{llllll}
\hline Day & Number of patients & & & \\
\cline { 2 - 6 } & Very much improved & Much improved & Improved & No change & Worse \\
\hline 30 & 1 & 2 & 2 & 2 & 0 \\
60 & 3 & 2 & 2 & 0 & 0 \\
120 & 4 & 2 & 1 & 0 & 0 \\
210 & 3 & 1 & 3 & 0 & 0 \\
\hline
\end{tabular}

Table 3 Subject satisfaction

\begin{tabular}{llll}
\hline Day & \multicolumn{2}{l}{ Number of patients } & Satisfied \\
\cline { 2 - 3 } & Very satisfied & & \\
30 & Q1: satisfaction with treatment & 0 \\
60 & 4 & 3 & 0 \\
210 & 6 & 1 & 0 \\
& 5 & 2 & \\
30 & Q2: appearance (telangiectasia, erythema, papules, etc.) after treatment & \\
60 & 3 & 3 & 0 \\
210 & 5 & 2 & 0 \\
30 & 5 & 2 & 0 \\
60 & Q3: symptoms (pruritus, burning or itching, dryness, etc.) remission & \\
210 & 2 & 5 & 0 \\
\hline
\end{tabular}

petechiae after injections was acceptable. The patients' judgment of appearance and symptoms after treatment changed with time; however, by termination of follow-up on day 210, all of the patients were still satisfied with the changes of their appearance and the improvement of the symptoms (Table 3 ).

\section{DISCUSSION AND CONCLUSION}

This dermatosis was given various names by different authors, such as steroid dermatitis resembling rosacea (SDRR), topical corticosteroid- induced rosacea-like dermatitis (TCIRD), topical steroid-induced facial rosaceiform dermatitis (TSIFRD), and steroid-induced rosacea-like dermatitis (SIRD) [1, 16, 20, 21]. Since there is no agreement on nomenclature, we prefer to use the term of corticosteroid-induced rosacea-like dermatitis (CIRD).

Patients with CIRD generally have telangiectasia, dryness, and burning or stinging sensation, indicating impaired skin barrier function, so restoration of the skin barrier function plays an important role in treatment. PRP is considered to contain growth factors that 
stimulate tissue repair, and percutaneous injection of PRP is used by some clinicians to treat epicondylitis, tendon and muscle injury, knee osteoarthritis, alopecia areata, and androgenetic alopecia [22-26]. In the last decade, PRP has been used more and more widely in dermatology, particularly in the areas of hair restoration [27-32], acne scars [33-35], dermal augmentation [36, 37], and striae distensae [38, 39]. Moreover, combination of PRP with laser treatments, microneedling, soft tissue fillers, and autologous fat grafting induces synergistic effects, leading to remarkable aesthetic results $[34,35,40,41]$. Therefore, we used PRP to treat CIRD through mesotherapy, intending to repair the skin barrier function as the preparation for any further treatment.

In addition to being an important source of molecules for tissue repair and regeneration, PRP has anti-inflammatory properties through its effects on the canonical nuclear factor- $\kappa \mathrm{B}$ signalling pathway in multiple cell types including synoviocytes, macrophages, and chondrocytes [42], so it has been used for treatment of sport-related injuries for several decades. However, there is a lack of high-quality evidence for the use of PRP for treatment of inflammatory skin disorders, and only a few case reports have shown that PRP can improve the symptoms of patients with atopic dermatitis and patients with chronic penile lichen sclerosus $[43,44]$. Therefore, the anti-inflammatory effect of PRP on CIRD and its mechanism need to be further studied.

The mesogun injector machine we used is controlled by a microcomputer. The amount of each injection, the injection speed, and the negative pressure intensity are set by computer parameters, which are easy to control. The negative pressure sucks up the skin during the injection, keeping the injected skin away from the blood vessels under the skin and reducing the risk of massive bleeding and hematoma. We injected PRP into the dermis with this mesogun, which not only guaranteed safety but also achieved a satisfactory therapeutic effect.

Mesotherapy is a general term describing a technique that involves multiple injections to deliver liquid preparations inclusive of medications and other substances into the subcutaneous tissue (the "mesoderm") to treat local medical and aesthetic conditions. It is minimally invasive and was accepted by all seven patients in our trial. Some patients reported more significant improvements in symptoms such as burning and dryness, while others showed more pronounced improvements in appearance such as telangiectasia and acneiform eruption, but overall each patient achieved satisfactory results. From the followup, the PRP repair effect is relatively lasting. We believe that when the skin barrier is functioning better, the subsequent treatment will respond faster. On the basis of these seven cases, we consider that it is worth conducting large randomized controlled trials to verify the safety and efficacy of PRP mesotherapy in treating CIRD. Plus, it may also provide new ideas for the treatment of rosacea and other skin disorders associated with impaired skin barrier function.

\section{ACKNOWLEDGEMENTS}

We thank the support and technical guidance from Shandong WEGO group medical polymer Co. Ltd, China.

Funding. No funding or sponsorship was received for this study. The journal's Rapid Service Fee was funded by Shaanxi Provincial Science and Technology Department (CN) received by Dr. Yue Yin. The award number is 2020 GXLH-Y-023.

Authorship. All named authors meet the International Committee of Medical Journal Editors (ICMJE) criteria for authorship for this article, take responsibility for the integrity of the work as a whole, and have given their approval for this version to be published.

Disclosures. Xing Fan, Yue Yin, Wenjie Dou, Tong Li, Ping Xue, Qing Yang and Qiaoxin Ma have nothing to disclose.

Compliance with Ethics Guidelines. We have obtained consent for publication from 
each subject with consent forms fully understood and signed by all the participants themselves. Written informed consent was obtained from the patient for publication of this case report and accompanying images.

Open Access. This article is licensed under a Creative Commons Attribution-NonCommercial 4.0 International License, which permits any non-commercial use, sharing, adaptation, distribution and reproduction in any medium or format, as long as you give appropriate credit to the original author(s) and the source, provide a link to the Creative Commons licence, and indicate if changes were made. The images or other third party material in this article are included in the article's Creative Commons licence, unless indicated otherwise in a credit line to the material. If material is not included in the article's Creative Commons licence and your intended use is not permitted by statutory regulation or exceeds the permitted use, you will need to obtain permission directly from the copyright holder. To view a copy of this licence, visit http:// creativecommons.org/licenses/by-nc/4.0/.

\section{REFERENCES}

1. Hameed AF. Steroid dermatitis resembling rosacea: a clinical evaluation of 75 patients. ISRN Dermatol. 2013;2013:491376.

2. Wilkin J. A role for vascular pathogenic mechanisms in rosacea: implications for patient care. Cutis. 2008;82(2):100.

3. Aksoy B, Altaykan-Hapa A, Egemen D, Karagöz F, Atakan N. The impact of rosacea on quality of life: effects of demographic and clinical characteristics and various treatment modalities. Br J Dermatol. 2010;163(4):719.

4. Crawford GH, Pelle MT, James WD. Rosacea: I. Etiology, pathogenesis, and subtype classification. J Am Acad Dermatol. 2004;51(3):327.

5. Ljubojeviae $\mathrm{S}$, Basta-Juzbasiae A, Lipozenèiae J. Steroid dermatitis resembling rosacea: aetiopathogenesis and treatment. J Eur Acad Dermatol Venereol. 2002;16(2):121-6.

6. Odom R, Dahl M, Dover J, et al. Standard management options for rosacea, part 2: options according to subtype. Cutis. 2009;84(2):97.
7. Pelle MT, Crawford GH, James WD. Rosacea: II. Therapy. J Am Acad Dermatol. 2004;51(4):499.

8. Tsunoda K, Akasaka K, Akasaka T, Amano H. Successful treatment of erythematotelangiectatic rosacea with intense pulsed light: report of 13 cases. J Dermatol. 2018;45(9):1113-6.

9. Seok J, Choi SY, Li K, Kim BJ, Kim MN, Hong CK. Recalcitrant steroid-induced rosacea successfully treated with $0.03 \%$ tacrolimus and $595-\mathrm{nm}$ pulsed dye laser. Eur J Dermatol. 2016;26(3):312-4.

10. Chen AY, Zirwas MJ. Steroid-induced rosacealike dermatitis: case report and review of the literature. Cutis. 2009;83(4):198-204.

11. Fujiwara S, Okubo Y, Irisawa R, Tsuboi R. Rosaceiform dermatitis associated with topical tacrolimus treatment. J Am Acad Dermatol. 2010;62(6):1050-2.

12. Eppley BL, Woodell JE, Higgins J. Platelet quantification and growth factor analysis from platelet-rich plasma: implications for wound healing. Plast Reconstr Surg. 2004;114(6):1502-8.

13. Sclafani AP, Romo T III, Ukrainsky G, et al. Modulation of wound response and soft tissue ingrowth in synthetic and allogeneic implants with platelet concentrate. Arch Facial Plast Surg. 2005;7:163-9.

14. Mishra A. Platelet-rich plasma. Orthopedics. 2010;33(7):486-7.

15. Chohan SN, Suhail M, Salman S, et al. Facial abuse of topical steroids and fairness creams: a clinical study of 200 patients. J Pak Assoc Dermatol. 2014;24(3):204-11.

16. Rathi SK, Kumrah L. Topical corticosteroid-induced rosacea-like dermatitis: a clinical study of 110 cases. Indian J Dermatol Venereol Leprol. 2011;77:42-6.

17. Hashim PW, Levy Z, Cohen JL, Goldenberg G. Microneedling therapy with and without plateletrich plasma. Cutis. 2017;99(4):239-42.

18. Wang HL, Avila G. Platelet rich plasma: myth or reality? Eur J Dent. 2007;1(4):192-4.

19. Kopera D, Palatin M, Bartsch R, et al. An open-label uncontrolled, multicenter study for the evaluation of the efficacy and safety of the dermal filler Princess VOLUME in the treatment of nasolabial folds. Biomed Res Int. 2015;2015:195328.

20. Tatu AL. Topical steroid induced facial rosaceiform dermatitis. Acta Endocrinol (Buchar). 2016;12(2): 232-3.

21. Wilkin J, Dahl M, Detmar M, et al. Standard grading system for rosacea: report of the National Rosacea 
Society Expert Committee on the classification and staging of rosacea. J Am Acad Dermatol. 2004;50(6): 907-12.

22. Arirachakaran A, Sukthuayat A, Sisayanarane T, et al. Platelet-rich plasma versus autologous blood versus steroid injection in lateral epicondylitis: systematic review and network meta-analysis. J Orthop Traumatol. 2016;17(2):101.

23. Fitzpatrick J, Bulsara M, Zheng MH. The effectiveness of platelet-rich plasma in the treatment of tendinopathy. Am J Sports Med. 2017;45(1):226.

24. Meheux CJ, McCulloch PC, Lintner DM, et al. Efficacy of intra-articular platelet-rich plasma injections in knee osteoarthritis: a systematic review. Arthroscopy. 2016;32(3):495.

25. Trink A, Sorbellini E, Bezzola P, et al. A randomized, double-blind, placebo- and active-controlled, halfhead study to evaluate the effects of platelet-rich plasma on alopecia areata. $\mathrm{Br} \mathrm{J}$ Dermatol. 2013;169(3):690-4.

26. Alves R, Grimalt R. Randomized placebo-controlled, double-blind, half-head study to assess the efficacy of platelet-rich plasma on the treatment of androgenetic alopecia. Dermatol Surg. 2016;42(4):491-7.

27. Sand JP, Nabili V, Kochhar A, Rawnsley J, Keller G. Platelet-rich plasma for the aesthetic surgeon. Facial Plast Surg. 2017;33(4):437-43.

28. Gupta AK, Carviel J. A mechanistic model of platelet-rich plasma treatment for androgenetic alopecia. Dermatol Surg. 2016;42(12):1335-9.

29. Cervelli V, Garcovich S, Bielli A, et al. The effect of autologous activated platelet-rich plasma (AA-PRP) injection on pattern hair loss: clinical and histomorphometric evaluation. BioMed Res Int. 2014;2014:760709.

30. Gkini MA, Kouskoukis AE, Tripsianis G, et al. Study of platelet-rich plasma inejctions in the treatment on androgenetic alopecia through an one-year period. J Cutan Aesthet Surg. 2014;7(4):213-9.

31. Takikawa M, Nakamura S, Nakamura S, et al. Enhanced effect of platelet-rich plasma containing a new carrier on hair growth. Dermatol Surg. 2011;37(12):1721-9.

32. Kang JS, Cheng Z, Choi MJ, et al. The effect of CD34+ cell-containing autologous platelet-rich plasma injection on pattern hair loss: a preliminary study. J Eur Acad Dermatol Venerol. 2014;28(1): 72-9.

33. Zhu JT, Xuan M, Zhang YN, et al. The efficacy of autologous platelet-rich plasma combined with erbium fractional laser therapy for facial acne scars or acne. Mol Med Rep. 2013;8(1):233-7.

34. Lee JW, Kim BJ, Kim MN, et al. The efficacy of autologous platelet rich plasma combined with ablative carbon dioxide fractional resurfacing for acne scars: a simultaneous split-face trial. Dermatol Surg. 2011;37(7):931-8.

35. Gawdat HI, Hegazy RA, Fawzy MM, et al. Autologous platelet-rich plasma: topical versus intradermal after fractional ablative carbon dioxide laser treatment of atrophic acne scars. Dermatol Surg. 2014;40(2):152-61.

36. Atkin DH, Trookman NS, Rizer RL, et al. Combination of physiologically balanced growth factors with antioxidants for reversal of facial photodamage. J Cosmet Laser Ther. 2010;12(1):14-20.

37. Kang BK, Shin MK, Lee JH, et al. Effects of plateletrich plasma on wrinkles and skin tone in Asian lower eyelid skin: preliminary results from a prospective, randomised, split-face trial. Eur J Dermatol. 2014;24(1):100-1.

38. Suh DH, Lee SJ, Lee JH, et al. Treatment of striae distensae combined enhanced penetration plateletrich plasma and ultrasound plasma fractional radiofrequency. J Cosmet Laser Ther. 2012;14(6): 272-6.

39. Kim IS, Park KY, Kim BJ, et al. Efficacy of intradermal radiofrequency combined with autologous platelet-rich plasma in striae distensae: a pilot study. Int J Dermatol. 2012;51(10):1253-8.

40. Na JI, Choi JW, Choi HR, et al. Rapid healing and reduced erythema after ablative fractional carbon dioxide laser resurfacing combined with the application of autologous platelet-rich plasma. Dermatol Surg. 2011;37(4):463-8.

41. Emer J. Platelet-rich plasma (PRP): current applications in dermatology. Skin Therapy Lett. 2019;24(5):1-6.

42. Andia I, Maffulli N. Platelet-rich plasma for managing pain and inflammation in osteoarthritis. Nat Rev Rheumatol. 2013;9(12):721-30.

43. Vafaei-Nodeh S, Kabiri-Abyaneh S. Long-term control of atopic dermatitis with platelet-rich plasma. JAAD Case Rep. 2020;5(7):54-6.

44. Casabona F, Gambelli I, Casabona F, Santi P, Santori $\mathrm{G}$, Baldelli I. Autologous platelet-rich plasma (PRP) in chronic penile lichen sclerosus: the impact on tissue repair and patient quality of life. Int Urol Nephrol. 2017;49(4):573-80. 